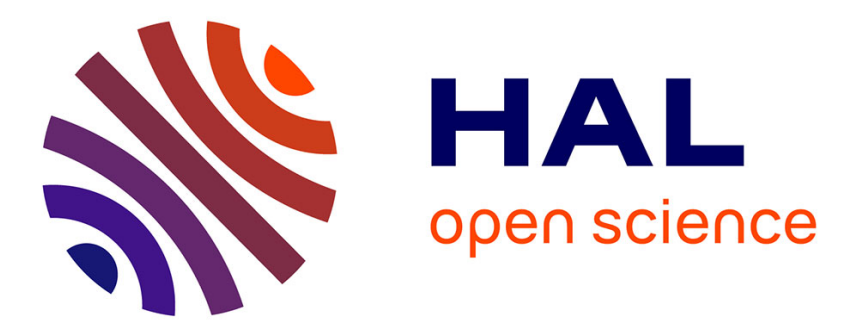

\title{
Microphase-separated tapered triblock copolymers
}

\author{
J. Samseth, R. Spontak, S. Smith, Arif Ashraf, K. Mortensen
}

\section{To cite this version:}

J. Samseth, R. Spontak, S. Smith, Arif Ashraf, K. Mortensen. Microphase-separated tapered triblock copolymers. Journal de Physique IV Proceedings, 1993, 03 (C8), pp.C8-59-C8-62. 10.1051/jp4:1993812 . jpa-00252243

\section{HAL Id: jpa-00252243 https://hal.science/jpa-00252243}

Submitted on 1 Jan 1993

HAL is a multi-disciplinary open access archive for the deposit and dissemination of scientific research documents, whether they are published or not. The documents may come from teaching and research institutions in France or abroad, or from public or private research centers.
L'archive ouverte pluridisciplinaire $\mathbf{H A L}$, est destinée au dépôt et à la diffusion de documents scientifiques de niveau recherche, publiés ou non, émanant des établissements d'enseignement et de recherche français ou étrangers, des laboratoires publics ou privés. 


\title{
Microphase-separated tapered triblock copolymers
}

\author{
J. SAMSETH, R.J. SPONTAK ${ }^{*}$, S.D. SMITH** ${ }^{*}$ A. ASHRAF ${ }^{* *}$ and K. MORTENSEN ${ }^{* * *}$ \\ Department of Physics, Institutt for Energiteknikk, Kjeller, Norway \\ "Department of Materials Science and Engineering, North Carolina State University, Raleigh, NC, U.S.A. \\ ** Corporate Research Division, The Procter and Gamble Company, Cincinnati, OH, U.S.A. \\ *** Department of Physics, Ris $\emptyset$ National Laboratory, Roskilde, Denmark
}

\begin{abstract}
The microphase-separated morphologies of a series of four poly[styrene- $b$-(d-styrene-gradisoprene)- $b$-isoprene] tapered block copolymers have been explored in this work with smallangle neutron scattering (SANS) and transmission electron microscopy (TEM). Each of the copolymers consists of two pure end blocks and a selectively deuterated statistical middle block, across which exists a composition gradient. The middle-block fraction varies from 10 to 40 $\mathrm{wt} \%$ in this series. All four materials are found to exhibit ordered lamellar morphologies, in which the microdomain periodicities do not differ significantly from that of the corresponding poly(styrene- $b$-isoprene) diblock copolymer of equal molecular weight $(\mathrm{M}=200 \mathrm{kDa})$. These results are compared with predictions from recent theoretical developments. In contrast, a 100 $\mathrm{kDa}$ poly[styrene- $b$-(styrene-ran-isoprene)- $b$-isoprene] triblock copolymer, possessing a $40 \mathrm{wt} \%$ statistical (random) middle block with an average composition of $50 \mathrm{wt} \%$ styrene, is observed to order into a lamellar-spherical three-microphase morphology upon block segregation.
\end{abstract}

\section{Introduction}

When the $\mathrm{A}$ and $\mathrm{B}$ blocks of an $\mathrm{AB}$ diblock copolymer are sufficiently incompatible, repulsive $\mathrm{A}-\mathrm{B}$ interactions dominate over the attractive $\mathrm{A}-\mathrm{A}$ and $\mathrm{B}-\mathrm{B}$ interactions, forcing the covalently bonded blocks to order into a periodic morphology. The magnitude of these interactions is described by $\chi N$, where $\chi$ is the Flory-Huggins parameter and $\mathrm{N}$ is the number of monomers along the chain. When $\chi N>>10$, diblock copolymers are safely removed from the order-disorder transition (ODT) [1] and reside within the strong-segregation regime. Experimental evidence [2-5] and theoretical predictions [6-10] indicate that $\chi$ scales as $1 / T$ (where $T$ denotes temperature) but also depends on monomer and block sequencing.

This latter factor warrants special consideration in the morphological behavior of tapered block copolymers. In tapered diblock copolymers, for instance, each block is composed of both A and B monomer species [11-14]. These copolymers undergo mixing within their microdomain cores and are capable of producing either ordered or disordered morphologies, depending on $\chi_{\text {eff }}[15]$. The magnitude of $\chi_{\text {eff }}$ can be estimated [16] from $\chi$ of the corresponding $A B$ diblock copolymer by

$$
\chi \text { eff } \approx \chi\left(\omega_{A}^{(A)}-\omega_{A}^{(B)}\right)^{2}
$$

where $\omega_{A}{ }^{(i)}$ is the fraction of $A$ in block $i$. Since $\chi_{\text {eff }}<\chi$ (due to enhanced attractive interactions) and since $\chi \sim 1 / T$, a tapered copolymer consequently exhibits a lower $\mathrm{T}_{\mathrm{ODT}}$ than does its $\mathrm{AB}$ diblock analogue.

Another class of tapered block copolymers, and the subject of the present work, includes triblock copolymers composed of pure A and B end blocks, along with a middle A/B block across which exists a composition gradient. Mixing in these copolymers is limited to the interphase region separating adjacent A and B microdomains. Previous morphological studies of both classes of tapered block copolymers [11-15] have revealed that incorporation of an A/B block within the copolymer can significantly affect the resultant morphology. In this work, we have employed small-angle neutron scattering (SANS) and transmission electron microscopy (TEM) to explore the morphological behavior of several microphaseseparated tapered triblock copolymers. 


\section{Experimental}

Four tapered triblock copolymers, composed of styrene (S) and isoprene (I) monomers, were synthesized via living anionic polymerization [13]. The S monomers in the middle S/I block were perdeuterated. Each tapered copolymer is designated here as TSI $\Phi$, where $\Phi$ denotes the weight percentage of the middle block. An additional copolymer, designated RSI40, was synthesized via a co-catalyzed reaction [15] so that the $40 \mathrm{wt} \% \mathrm{~S} / \mathrm{I}$ block possessed a constant mean composition $(50 \mathrm{wt} \% \mathrm{~S})$ rather than a composition gradient. The composition of each material was determined to be about $50 \mathrm{wt} \% \mathrm{~S}$ with ${ }^{1} \mathrm{H}$ NMR. Molecular weights, discerned with GPC and expressed in $\mathrm{kDa}$, varied within the tapered copolymer series: 204 (TSI 10); 259 (TSI20); 162 (TSI30); and 274 (TSI40). The RSI40 specimen possessed a molecular weight of about $100 \mathrm{kDa}$. Polydispersities were less than 1.10 in all cases.

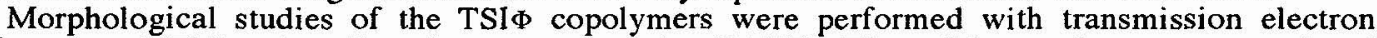
microscopy (TEM) and small-angle neutron scattering (SANS). Films of the copolymers were produced from $5 \%(\mathrm{wt} / \mathrm{v})$ solutions in toluene. For the TSI\$ copolymers, the rate of solvent evaporation was not controlled, and resultant films were annealed at $100^{\circ} \mathrm{C}$ to remove residual solvent. The RSI40 film was produced using the protocol described in detail elsewhere [17].

TEM specimens were prepared by sectioning each film normal to the film surface at $-100^{\circ} \mathrm{C}$. The sections were subsequently stained with $\mathrm{OsO}_{4}$ vapor and examined on a Zeiss EM902 electron spectroscopic microscope, operated at $80 \mathrm{keV}$ and $\Delta \mathrm{E} \approx 125 \mathrm{eV}$. Two-dimensional SANS patterns of the TSI $\Phi$ copolymers only were acquired both normal and parallel to the surface of each cast film with the ${ }^{3}$ He detector at Risø National Laboratory. The sample-to-detector distance was $6.0 \mathrm{~m}$, and the neutron wavelength $(\lambda)$ was $1.02 \mathrm{~nm}$. The patterns were corrected for absorption and dark current, and they were subsequently normalized with respect to water and integrated.

\section{Results and Discussion}

Figure 1 consists of a series of electron micrographs illustrating the microphase-separated morphologies present in the four TSI $\Phi$ copolymers. Lamellar morphologies, anticipated from the known copolymer compositions, are evident in each. The lamellae in TSI 10 (Fig. 1a) appear to be well-oriented and are reminiscent of conventional SI diblock copolymers. As $\Phi$ increases from $10 \mathrm{wt} \%$ (Fig. 1a) to 40 wt\% (Fig. 1d), though, the oriented lamellar grains are observed to decrease in size, while thin styrenerich lamellae appear. These thin, thread-like microdomains are especially visible in micrographs of both the TSI 20 and TSI 40 copolymers (Figs. $1 \mathrm{~b}$ and $1 \mathrm{~d}$, respectively). While lamellar morphologies exhibit a variety of different grain boundaries [17], this transition from well-ordered lamellae to thin microdomains has not, to the best of our knowledge, been previously reported in diblock copolymers. It is, however, consistent with the reduction in the length of the S block that accompanies an increase in $\Phi$.

While not shown here, SANS patterns obtained from each specimen with its film surface oriented normal to the neutron beam appear isotropic. When the film surface is oriented parallel to the neutron beam, the resultant pattern for every TSI $\Phi$ copolymer consists of arcs. Such anisotropic scattering patterns are characteristic of the lamellar morphology, and corresponding intensity profiles are provided in Fig. 2 as a function of the magnitude of the scattering vector $\mathrm{q}$, defined by

$$
q=\frac{4 \pi}{\lambda} \sin \frac{\theta}{2}
$$

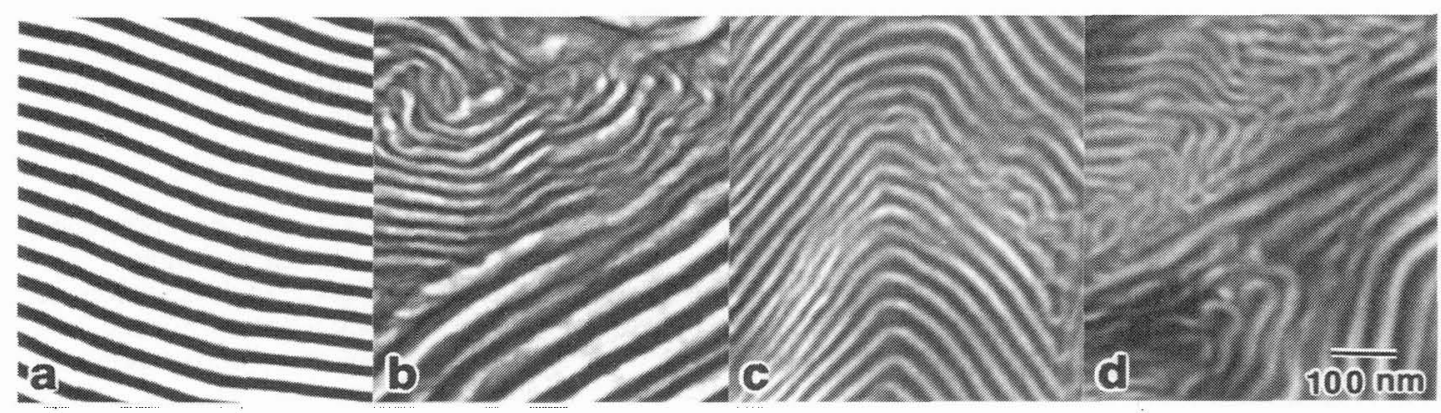

Fig. 1. TEM micrographs of the four TSI $\Phi$ copolymers possessing different $\Phi$ (in wt\%): (a) 10, (b) 20 , (c) 30 and (d) 40 . Isoprene microdomains appear dark due to the preferential $\mathrm{OsO}_{4}$ stain. 


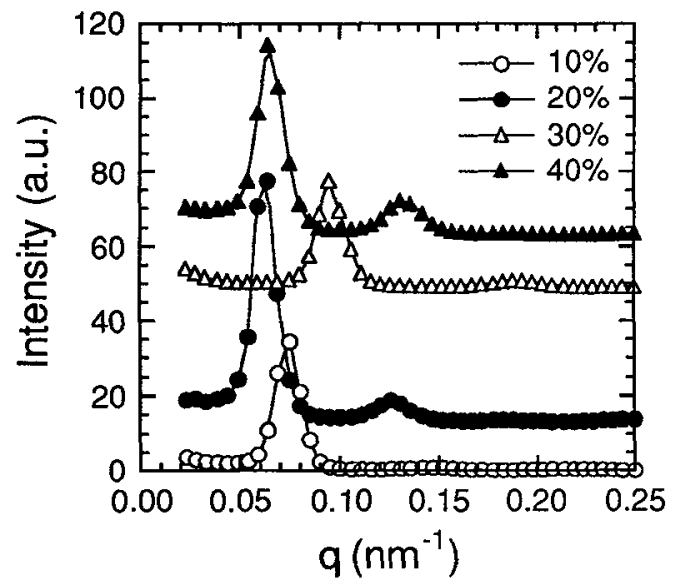

Fig. 2. Scattering curves as a function of $q$ for the four TSI $\Phi$ copolymers. The top curves have been shifted up to facilitate peak discrimination.

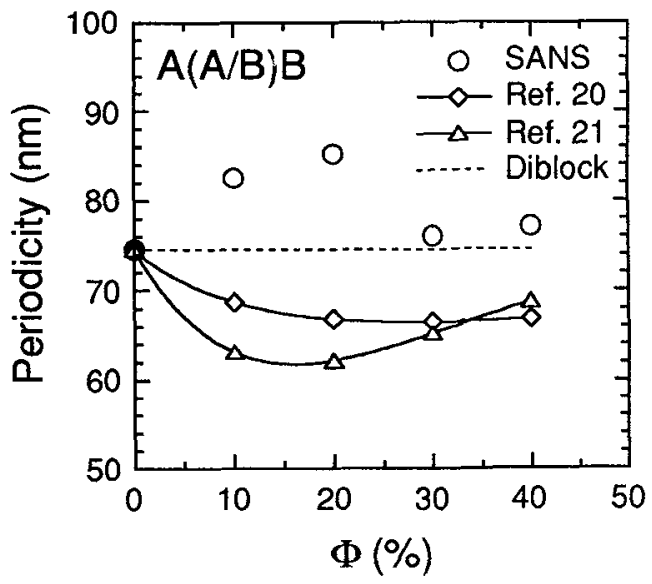

Fig. 3. Functional relationship between $D_{c}(o)$ and $\Phi$ showing little change in $\mathrm{D}_{\mathrm{c}}$ from $\mathrm{D}_{\mathrm{c}}(\Phi=0)$. Predicted $D_{c}$ are shown as labeled solid lines.

where $\theta$ is the scattering angle. Each curve in Fig. 2 exhibits a scattering maximum at position $q^{*}$ and another minor peak at $2 q^{*}$, indicative of long-range order. A characteristic periodicity (D) can be obtained from $\mathrm{q}^{*}$ through Bragg's law, i.e., $\mathrm{D}=2 \pi / \mathrm{q}^{*}$. Values of $\mathrm{D}$ in this study correspond to the microdomain periodicity and not to the repeat distance of the deuterated S/I middle block (due to the $16 \%$ difference in size between the S and I lamellae). The D measured here are, however, complicated by the variation in chain length among the four copolymers. In strongly-segregated $\mathrm{AB}$ diblock copolymers, $\mathrm{D} \sim \mathrm{M}^{2 / 3}$. Recent theoretical developments addressing the microstructural characteristics of both strongly-segregated $\mathrm{ABC}[18-20]$ and $\mathrm{A}(\mathrm{A} / \mathrm{B}) \mathrm{B}[20,21]$ architectures indicate that this scaling behavior is retained in linear triblock terpolymers and copolymers, respectively. Consequently, we can correct the $\mathrm{D}$ measured here by defining $\mathrm{D}_{\mathrm{c}}$ such that

$$
\mathrm{D}_{\mathrm{c}}=\mathrm{D}\left(\frac{\mathrm{M}^{\mathrm{o}}}{\mathrm{M}}\right)^{2 / 3}
$$

where $\mathrm{M}^{\circ}=200 \mathrm{kDa}$. Upon doing so, the functional relationship of $\mathrm{D}_{\mathrm{c}}(\Phi)$ can be obtained (see Fig. 3). The value of $D_{c}$ at $\Phi=0$ is extrapolated, via Eq. (3), from scattering data obtained in a prior study of SI diblock copolymers by Hashimoto et al. [22]. It is clear from Fig. 3 that the periodicity of these tapered triblock copolymers does not differ substantially from that of the diblock analogue. In fact, within the scatter of the data, no clearly discernible trend is observed. The lack of an unambiguous $D_{c}(\Phi)$ relationship may reflect variation in the compositional gradient across the middle S/I block [15]. A recent extension [20] of the Semenov [23] formalism (for strongly segregated diblock copolymers) to $\mathrm{A}(\mathrm{A} / \mathrm{B}) \mathrm{B}$ copolymers has yielded the following expression for the equilibrium microdomain periodicity:

$$
\mathrm{D}_{\mathrm{A}(\mathrm{A} / \mathrm{B}) \mathrm{B}}=\frac{\mathrm{D}_{\mathrm{AB}}(1+f)}{(1+7 f)^{1 / 3}}
$$

where $\mathrm{f}$ is the monomer fraction of the middle $\mathrm{A} / \mathrm{B}$ block $(\approx \Phi / 100)$ and $\mathrm{D}_{\mathrm{AB}}$ is the periodicity of the corresponding diblock copolymer (as $\mathrm{f} \rightarrow 0$ ), which is proportional to $\mathrm{aN}^{2 / 3} \chi^{1 / 6}$ ( $\mathrm{a}$ is the monomer length). Equation 4 implicitly depends on the validity of Eq. 1, which does not account for a gradient (or isotopic labeling) across the middle A/B block. Predictions for the microdomain periodicity obtained from Eq. 4, as well as from a confined single-chain approach described elsewhere [21], are also shown in Fig. 3.

An electron micrograph of the RSI40 copolymer is provided in Fig. 4. Recall that it is comparable to TSI40 except for the absence of a compositional gradient across the middle S/I block. The morphology seen here is the "spherical-lamellar" morphology, first reported by Hashimoto et al. [11] for a tapered diblock copolymer. This is a three-microphase morphology in which the short lamellae, constrained spheres, and matrix are composed of the S, I, and S/I blocks, respectively. The appearance of this morphology in this copolymer strongly suggests that the copolymer synthesized earlier [11] was not a tapered diblock copolymer. It is obvious that this morphology does not resemble that seen in Fig. 1d, indicating that monomer sequencing along the middle block plays an important role in the development of ordered microstructures in $\mathrm{A}(\mathrm{A} / \mathrm{B}) \mathrm{B}$ triblock copolymers. 


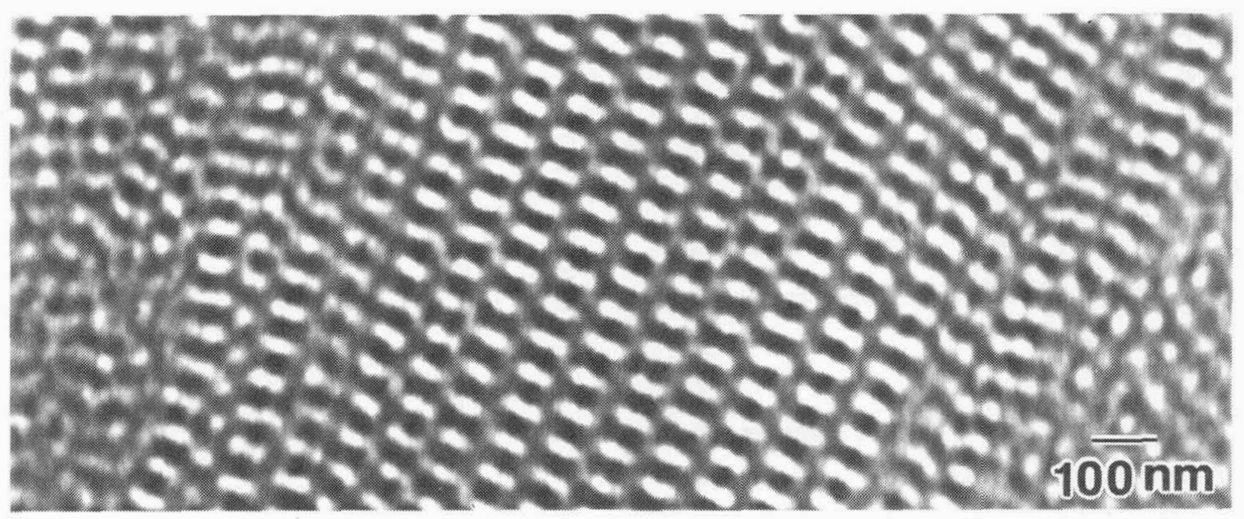

Fig. 4. Electron micrograph of the RSI40 copolymer illustrating the three-microphase "sphericallamellar" morphology, in which $S$ appears light, S/I appears darker, and I appears the darkest. The difference between this copolymer and TSI 40 , in addition to molecular weight, lies in the fact that the S/I block in the RSI 40 copolymer does not possess a composition gradient.

\section{Concluding Remarks}

We have investigated the microstructural characteristics of a series of tapered triblock copolymers with SANS and TEM. Each copolymer exhibits a lamellar morphology. As $\Phi$ increases, TEM micrographs reveal that the oriented lamellae undergo a transition to disjoint thin lamellae, which might signal the onset of either a three-microphase morphology or microstructural disorder. Microdomain periodicities, obtained with SANS, are virtually independent of $\Phi$. If the middle block is statistical (i.e., without a composition gradient), a "spherical-lamellar" three-microphase morphology is capable of developing rather an alternating lamellar morphology.

\section{References}

[1] Leibler, L., Macromolecules 13 (1980) 1602.

[2] Owens, J.N., Gancarz, I.S., Koberstein, J.T. and Russell, T.P., Macromolecules 22 (1989) 3380.

[3] Koberstein, J.T., Russell, T.P. and Walsh, D.J., Macromolecules 23 (1990) 877.

[4] Gehlsen, M.D., Almdal, K. and Bates, F.S., Macromolecules 25 (1992) 939.

[5] Samseth, J., Mortensen, K., Burns, J.L. and Spontak, R.J., J. Appl. Polym. Sci. 44 (1992) 1245.

[6] Mayes, A.A. and Olvera de la Cruz, M., J. Chem. Phys. 91 (1989) 7228.

[7] Benoit, H. and Hadziioannou, G., Macromolecules 21 (1988) 1449.

[8] Kavassalis, T. and Whitmore, M.D., Macromolecules 24 (1991) 5340.

[9] Fredrickson, G.H. and Milner, S.T., Phys. Rev. Lett. 67 (1991) 835.

[10] Fredrickson, G.H., Milner, S.T. and Leibler, L., Macromolecules 25 (1992) 6341.

[11] Hashimoto, T., Tsukahara, Y., Tachi, K. and Kawai, H., Macromolecules 16 (1983) 648.

[12] Tsukahara, Y., Nakamura, N., Hashimoto, T. and Kawai, H., Polym. J. 12 (1980) 455.

[13] Annighöfer, F. and Gronski, W., Colloid Polym. Sci. 261 (1983) 15; Makromol. Chem. 185 (1984) 2213.

[14] Buihler, F. and Gronski, W., Makromol. Chem. 187, 2019 (1986); 188, 2995 (1987); 189 (1988) 1087.

[15] Ashraf, A., Smith, S.D. and Clarson, S.J., Macromolecules (submitted).

[16] Hashimoto, T., in Thermoplastic Elastomers: A Comprehensive Review (N.R. Legge, G. Holden and H.E. Schroeder, eds.) Hanser, Munich, 1987, Chap. 12.

[17] Spontak, R.J., Smith, S.D. and Ashraf, A., Macromolecules 26 (1993) 956.

[18] Spontak, R.J. and Zielinski, J.M., Macromolecules 25 (1992) 663.

[19] Nakazawa, $H$. and Ohta, T., Macromolecules (in press).

[20] Kane, L.L. and Spontak, R.J., Macromolecules (submitted).

[21] Zielinski, J.M. and Spontak, R.J., Macromolecules 25 (1992) 5957.

[22] Hashimoto, T., Shibayama, M. and Kawai, H., Macromolecules 13 (1980) 1237.

[23] Semenov, A.N., Sov. Phys. JETP 61 (1985) 733. 